doi:10.17659/01.2019.0056

Journal of Case Reports 2019;9(4):211-213

\title{
Wernicke's Encephalopathy Presenting as Hyperemesis Gravidarum: Keys to a Successful Outcome
}

\author{
Nishtha Jaiswal, Reena Yadav, Astha Srivastava \\ Department of Obstetrics and Gynaecology, SSK Hospital and Lady Hardinge Medical College, New Delhi, India-110001.
}

\section{Corresponding Author: \\ Dr Nishtha Jaiswal \\ Email: nishtha.amu@gmail.com}

This is an Open Access article distributed under the terms of the Creative Commons Attribution License (creativecommons.org/ licenses/by/3.0).

Received Accepted Published

July 9, 2019

September 1, 2019

October 15, 2019

\begin{abstract}
Background: Wernicke's encephalopathy (WE) is a rare condition resulting from chronic malnutrition. The commonest cause of WE is chronic alcoholism. Hyperemesis in pregnancy is another overlooked condition that can result in chronic malnutrition and can precipitate WE. WE in pregnancy leads to poor outcomes both for the mother and the baby. It is a rare condition and needs a high degree of suspicion for diagnosis. Case Report: A 27 years old pregnant lady presented to our hospital in altered sensorium. After excluding the common causes of encephalopathy in pregnancy, WE was considered due to a history of hyperemesis. The MRI of brain supported the diagnosis. The woman was started on thiamine and had a gradual improvement in her general condition. She went on to successfully complete the pregnancy and delivered a healthy baby. Conclusion: Early recognition of this condition and prompt thiamine administration was the key to successful outcome.
\end{abstract}

Keywords: Brain, Hyperemesis Gravidarum, Pregnancy, Thiamine, Wernicke's Encephalopathy.

\section{Introduction}

Wernicke's encephalopathy (WE) is a result of thiamine deficiency secondary to conditions like chronic alcoholism or hyperemesis in pregnancy. It presents with neuropsychiatric symptoms. The outcomes in untreated WE in pregnancy are poor both for the mother and the fetus. Timely detection based on clinical features and imaging is important to initiate therapy. Here we report a 27 years old pregnant patient with a history of hyperemesis gravidarum. She presented with altered sensorium in her second trimester and was diagnosed with WE. This case will add to the few available in literature where a rare diagnosis of WE was suspected in a pregnant patient with hyperemesis. The successful maternal and fetal outcome in this case with timely therapy also makes it worth reporting.

\section{Case Report}

A 27 years old pregnant lady presented to the emergency at 16 weeks of gestation with complaints of altered sensorium and urinary incontinence for five days. She had one living issue and had two abortions. She had a history of excessive vomiting (hyperemesis gravidarum) since the first month of gestation resulting in cachexia. The patient was unconscious but hemodynamically stable. The blood pressure was 120/80 mm Hg. The systemic examination of the patient revealed the presence of neck rigidity, hypotonia in the limbs and upgoing plantar reflexes bilaterally. Her blood and urine investigations were normal. The ultrasound of the abdomen showed a single live intrauterine fetus of 15 weeks gestation. 
The patient was intubated at admission because of poor sensorium and was started on supportive management. Later she developed ventilator associated pneumonia for which she required broad spectrum antibiotics. The antinuclear antibody titres and cerebrospinal (CSF) examination were within normal limits. The magnetic resonance imaging (MRI) of the brain revealed symmetrical T2 flair hyper-intensities in bilateral thalami, midbrain and medulla with variable diffusion restriction, and the possibility of encephalitis was considered [Fig.1].

A diagnosis of WE was considered and the patient was started on intravenous (IV) thiamine injections at a dose of $100 \mathrm{mg}$ IV twice a day. The patient had to be tracheostomised for prolonged ventilation. She showed improvement in her sensorium and was weaned off the ventilator on the $32^{\text {nd }}$ day of admission. She was started on oral thiamine at a dose of $100 \mathrm{mg}$ thrice daily via the nasogastric tube initially and then orally when she could swallow the tablets. She showed improvement on the above management and regained her sensorium. The patient was discharged after 68 days at 23 weeks of gestation. She went on to complete her pregnancy and delivered a healthy male baby weighing $2.25 \mathrm{~kg}$ at 36 weeks of gestational age. The mode of delivery was an elective cesarean section.

\section{Discussion}

WE presents with neuropsychiatric symptoms and is caused by thiamine (vitamin-B1) deficiency. Malnutrition and consequent vitamin B1 deficiency can precipitate it [1]. Hyperemesis gravidarum during pregnancy is one of such causes. It can be responsible for neuropsychiatric symptoms in pregnant patients and if untreated this can result in fatal outcomes both for the mother and the fetus [2]. As per the European Federation of Neurological Societies guidelines WE requires two of the following four criteria for diagnosis: dietary deficiency, eye signs (nystagmus), cerebellar
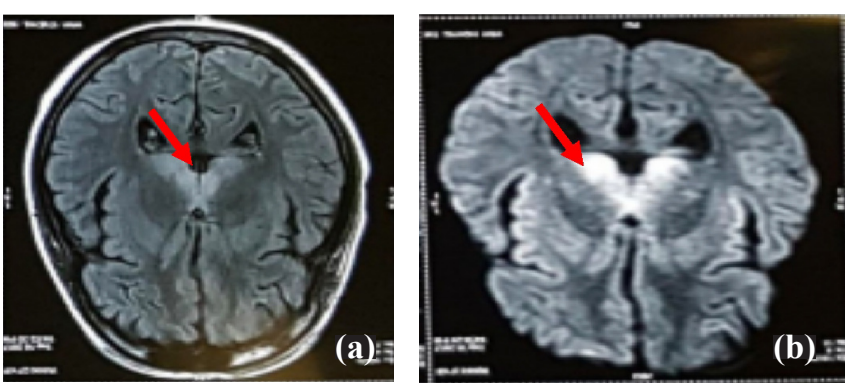

Fig.1: MRI brain of patient showing symmetrical T2 flair hyper-intensities in bilateral thalami, midbrain and medulla.

dysfunction, and either an altered mental state or mild memory impairment [3]. Improvement in symptoms following thiamine administration confirms the diagnosis retrospectively.

MRI of the brain has a high sensitivity and specificity for detecting changes in WE but a normal MRI cannot rule out WE. Bilateral and symmetrical hyper-intense lesions on T2-weighted and FLAIR sequences are observed in peri-aqueductal gray matter, thalamus, mammillary bodies and around the third-ventricle [4]. Hyperemesis gravidarum is known to be associated with poor pregnancy outcomes like low birth weight, intra-uterine growth retardation and risk of premature birth [5]. Thiamine deficiency can adversely affect fetal growth resulting in intrauterine growth retardation. Fetal outcomes in WE are poor and mortality has been reported in upto $40 \%$ [6].

WE should be treated with 100-200 mg of parenteral thiamine hydrochloride three times a day, diluted in $100 \mathrm{~mL}$ of normal saline or $5 \%$ dextrose. If WE is suspected plain dextrose infusions without concomitant thiamine should be avoided as it can further precipitate thiamine deficiency. $100 \mathrm{mg}$ of oral thiamine should be given up to three times a day after discharge until normal diet intake is achieved [7]. It is also advisable to supplement thiamine in patients with hyperemesis during pregnancy.

\section{Conclusion}

It is important to consider WE as one of the causes of altered sensorium in pregnant patient especially 
with a background of malnutrition or hyperemesis. A timely initiation of treatment in this case led to improvement in her condition and favourable pregnancy outcome.

Contributors: NJ: manuscript writing and editing, patient management; RY, AS: patient management, critical inputs into the manuscript. NJ will act as a study guarantor. All authors have seen and approved the final version of this manuscript.

Funding: None; Competing interests: None stated.

\section{References}

1. Sechi G, Serra A. Wernicke's encephalopathy: new clinical settings and recent advances in diagnosis and management. Lancet Neurol. 2007;6:442-455.

2. Williams NL, Wiegand S, McKenna DS. Wernicke's encephalopathy complicating pregnancy in a woman with neonatal necrotizing enterocolitis and resultant chronic malabsorption. Am J Perinatol. 2009;26:519521.

3. Galvin R, Bråthen G, Ivashynka A, Hillbom M, Tanasescu R, Leone MA. EFNS guidelines for diagnosis, therapy and prevention of Wernicke encephalopathy. Eur J Neurol. 2010;17:1408-1418.

4. Thomson AD, Marshall EJ. The natural history and pathophysiology of Wernicke's encephalopathy and Korsakoff's psychosis. Alcohol. 2006;41(2):151-158.

5. Veenendaal MV, van Abeelen AF, Painter RC, van der Post JA, Roseboom TJ. Consequences of hyperemesis gravidarum for o spring: a systematic review and metaanalysis. Br J Obstet Gynaecol. 2011;118:1302-1313.

6. Selitsky T, Chandra P, Schiavello HJ. Wernicke's encephalopathy with hyperemesis and ketoacidosis. Obstet Gynecol. 2006;107:486-490.

7. Thomson AD, Guerrini I, Marshall EJ. Wernicke's encephalopathy: role of thiamine. Pract Gastroenterol. 2009;23:21-30. 